RELATO DE CASOS

\title{
Uso de Terapia Biológica na Doença de Crohn Metastática: Relato de Caso e Revisão da Literatura
}

\author{
Use of Biological Therapy in Metastatic Crohn's Disease: \\ A Case Report and Literature Review
}

\section{RODRIGO ROCHABATISTA ${ }^{1}$; BÁRBARA HELOISAZANCHETTAPOZZOBON ${ }^{1}$; JULIANAMAGALHÃES LOPES ${ }^{2}$; IDBLANCARVALHODEALBUQUERQUE ${ }^{3}$; GALDINO JOSÉ SITONIO FORMIGA ${ }^{4}$}

\footnotetext{
${ }^{1}$ Residente de Coloproctologia do Hospital Heliópolis; ${ }^{2}$ Ex-residente de Coloproctologia do Hospital Heliópolis; ${ }^{3}$ Médico Assistente do Serviço de Coloproctologia do Hospital Heliópolis; ${ }^{4}$ Chefe do Serviço de Coloproctologia do Hospital Heliópolis.
}

BATISTA RR; POZZOBON BHZ; LOPES JM; ALBUQUERQUE IC; FORMIGA GJS. Uso de Terapia Biológica na Doença de Crohn Metastática: Relato de Caso e Revisão da Literatura. Rev bras Coloproct, 2009;29(3): 372-376.

RESUMO: A doença de Crohn metastática envolve a infiltração cutânea granulomatosa em locais anatomicamente separados do trato gastrointestinal, com tendência à cronicidade. É relatado caso de paciente masculino, 20 anos, há seis meses com dor e eliminação de secreção purulenta de úlceras em região perianal, inguinal direita e genital, sem melhora com o uso metronidazol e ciprofloxacina. Antecedente de proctocolectomia em 2002. Ao exame, à inspeção, evidenciava-se orifício fistuloso posterior a 2,0 cm da borda anal, úlcera de $5,0 \mathrm{~cm}$ na base do escroto e outra úlcera circundando a base do pênis; ao toque, ânus fibrótico e encarcerado. Realizada fistulotomia, biópsia e curetagem das úlceras genital e inguinal. $O$ resultado histopatológico evidenciou processo inflamatório granuloso não caseoso. Em virtude da falha terapêutica dos antimicrobianos, foi optado pelo tratamento com infliximabe na dose $5 \mathrm{mg} / \mathrm{kg}$ nas semanas 0,2 e 6, e azatioprina $2 \mathrm{mg} / \mathrm{kg} / \mathrm{dia}$. Ao término da fase de indução, o paciente apresentava cicatrização parcial das lesões ulceradas, ausência de secreção e alívio da dor. Atualmente em acompanhamento ambulatorial com infusões de infliximabe a cada oito semanas.

Descritores: Doença de Crohn; Metastática; Pele; Períneo; Terapia biológica.

\section{INTRODUÇÃO}

A doença de Crohn (DC) é uma doença inflamatória intestinal de evolução crônica e recidivante, caracterizada por inflamação transmural segmentar e alterações granulomatosas, que pode envolver qualquer segmento do trato gastrointestinal. ${ }^{(1-9)}$ Foi descrita em 1932 por Crohn, Ginzberg e Oppenheimer, e originalmente chamada de ileíte regional. ${ }^{(10)}$ Os pacientes podem desenvolver manifestações extra-intestinais em 25 a $30 \%$ dos casos, principalmente cutâneas, as quais ocorrem em 14-44\% dos casos, dependendo se a doença perineal é ou não considerada uma manifestação cutânea. ${ }^{(1,3,8,11)}$

A doença de Crohn metastática (DCM) envolve a infiltração cutânea granulomatosa em locais anatomicamente separados do trato gastrointestinal, com tendência à cronicidade. ${ }^{(1-6,9,11-14)}$ Foi primeiramente descrita por Parks AG et al. em 1965. ${ }^{(17)}$ Em 1970, o termo ulceração metastática foi adotado por Mountain JC para descrever as úlceras cutâneas que surgiam distantes da região perianal ou periostomal. ${ }^{(18)}$

As terapias convencionais têm resultado pouco satisfatório na $\mathrm{DCM} .^{(2,15)}$ A terapia biológica com 
infliximabe apresenta resposta clínica satisfatória com baixo índice de complicações nestes pacientes. O objetivo deste relato foi apresentar um caso de doença de Crohn metastática em região inguinal e genital tratada com infliximabe.

\section{RELATO DO CASO}

L.A.D.F., 20 anos, masculino, branco, estudante. Há seis meses com queixa de dor e eliminação de secreção purulenta de úlceras em região perianal, inguinal direita e genital, sem melhora com o uso metronidazol e ciprofloxacina. Antecedente cirúrgico de proctocolectomia em 2002 por DC colônica. Ao exame físico geral sem alterações.

No exame proctológico e pelve-perineal sob anestesia, à inspeção, evidenciava-se orifício fistuloso posterior a 2,0 cm da borda anal (Figura 1a), úlcera de $5,0 \mathrm{~cm}$ na base do escroto (Figura 1b) e outra úlcera circundando a base do pênis (Figura 1c); ao toque, ânus fibrótico e encarcerado. Realizada fistulotomia, biópsia e curetagem das úlceras genital e inguinal. O resultado histopatológico evidenciou processo inflamatório granuloso não caseoso.

Em virtude da falha terapêutica dos antimicrobianos, foi optado pelo tratamento com infliximabe na dose $5 \mathrm{mg} / \mathrm{kg}$ nas semanas 0,2 e 6 , associado a azatioprina $2 \mathrm{mg} / \mathrm{kg} / \mathrm{dia}$. Ao término da fase de indução, o paciente apresentava cicatrização parcial das lesões ulceradas, ausência de secreção e alívio da dor. Atualmente em acompanhamento ambulatorial com infusões de infliximabe a cada oito semanas e com cicatrização completa das lesões (Figuras 2a e 2b).

\section{DISCUSSÃO}

As manifestações cutâneas da doença de Crohn são comuns e podem ser divididas em três tipos distintos. O mais comum é a úlcera ou a fístula perianal e periostomal, na qual o processo inflamatório da DC no trato gastrointestinal envolve a pele adjacente. $\mathrm{O}$ segundo tipo inclui manifestações cutâneas secundárias à deficiência nutricional e reativas (imuno-relacionadas, porém sem componente granulomatoso), tais como acrodermatite enteropática (deficiência adquirida de zinco), pioderma gangrenoso, eritema nodoso, eritema multiforme, epidermólise bolhosa, amiloidose sistêmica secundária, vitiligo, pustulose palmo-plantar

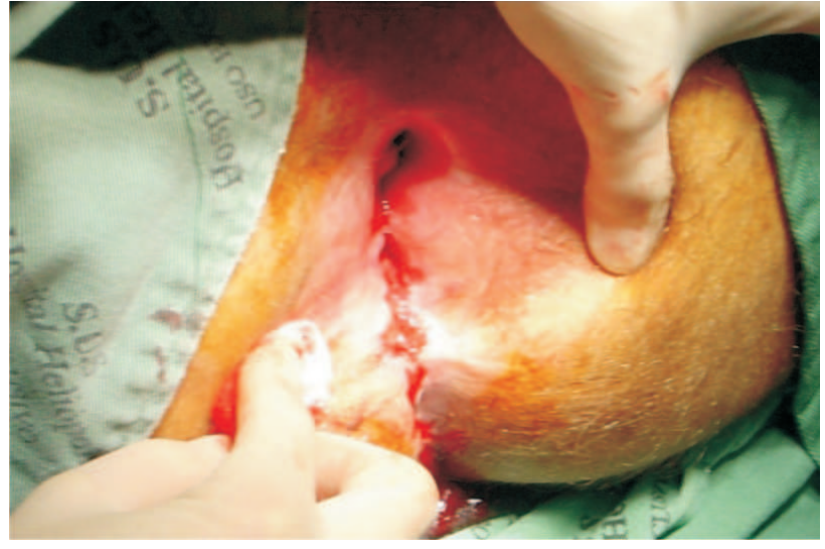

Figura 1a - Inspeção anal.

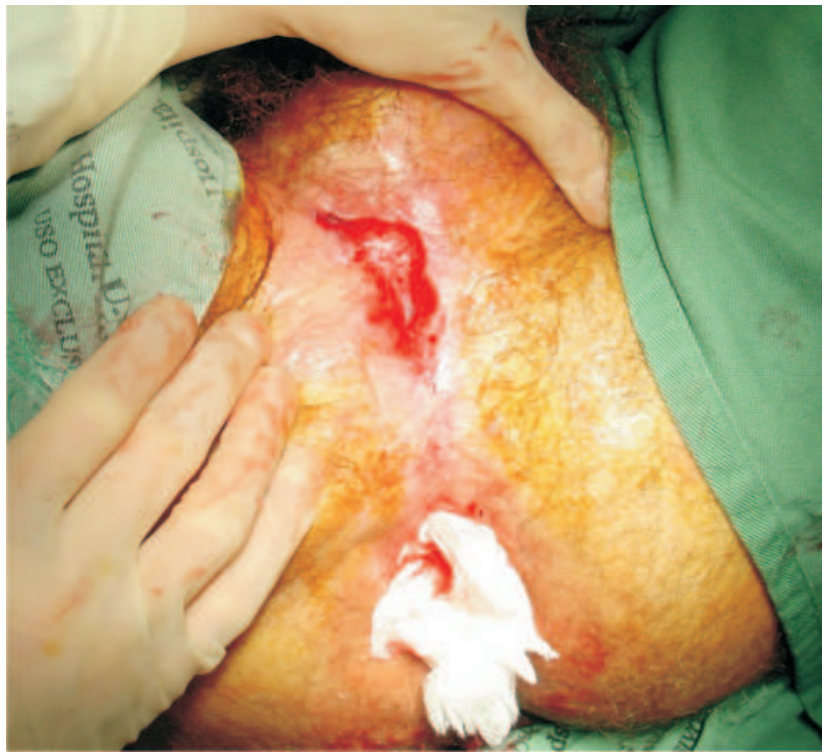

Figura $1 b$ - Úlcera na base do escroto.

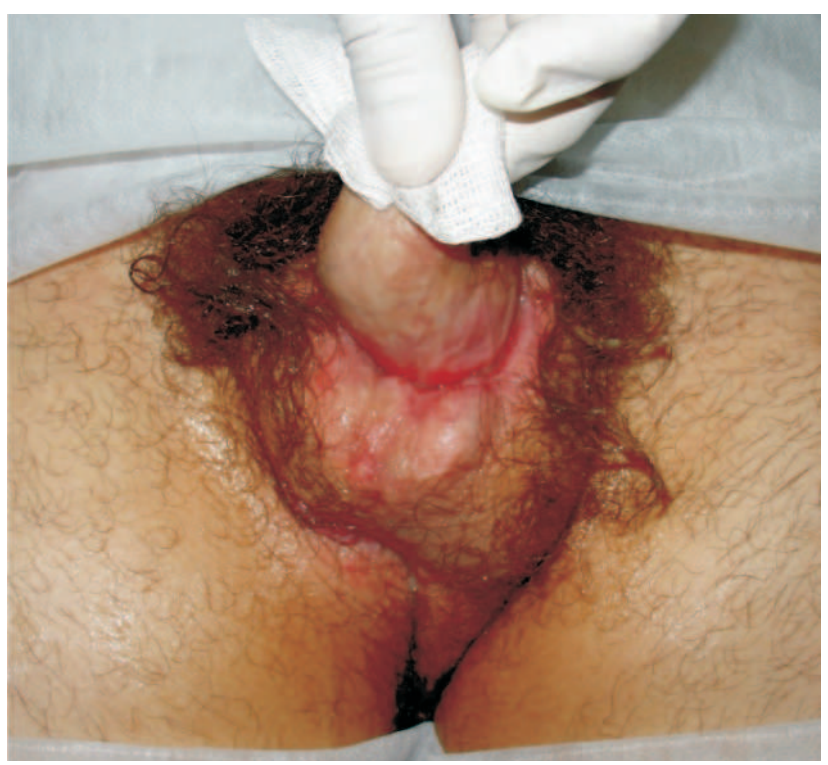

Figura 1c - Úlcera na base do pênis. 


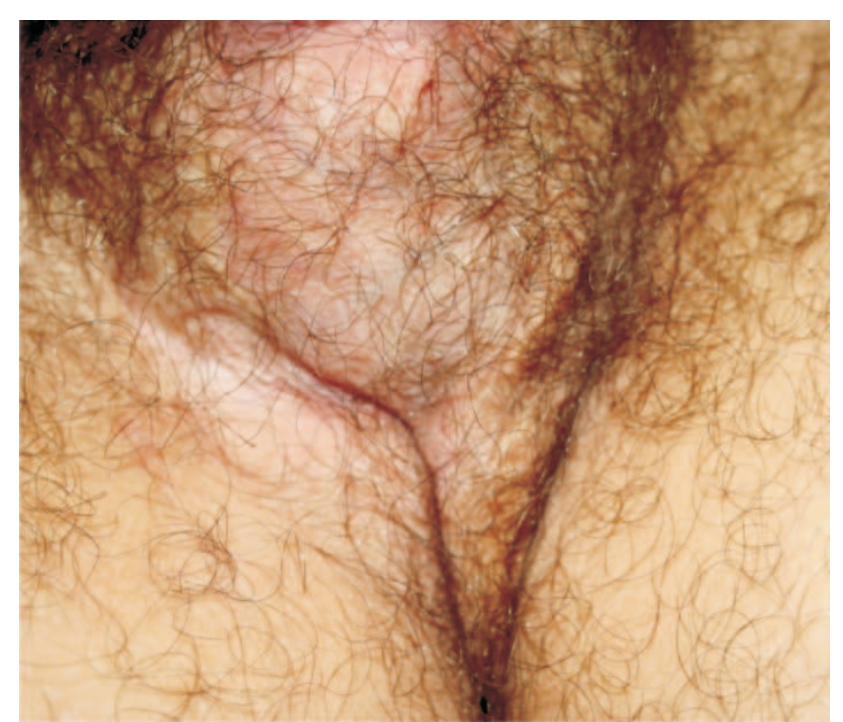

Figura 2a - Cicatriz na base da bolsa escrotal.

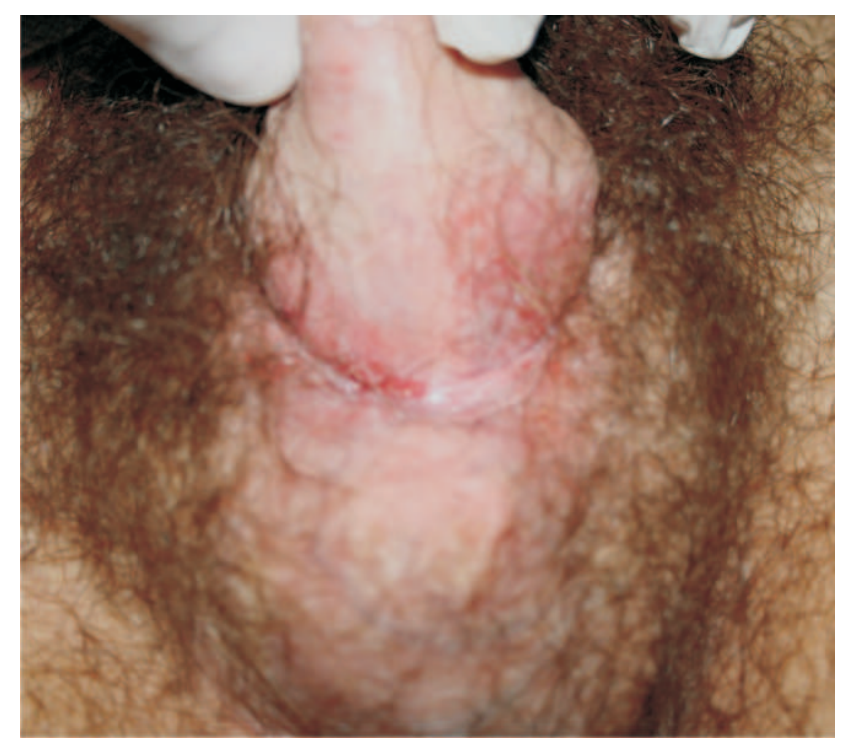

Figura $2 \boldsymbol{b}$ - Base do pênis, após seis meses de infliximabe.

e síndrome de Sweet. O terceiro tipo, mais raro, a DCM, consiste em lesões cutâneas granulomatosas sem contiguidade com o trato gastrointestinal. . $2-5,8,12,14-16)^{-}$

A patogênese da DCM ainda é pouco entendida. Em 1981, Burgdorf W sugeriu que imunocomplexos depositados na pele seriam responsáveis pela reação granulomatosa. ${ }^{(19)} \mathrm{Um}$ segundo mecanismo sugerido, foi de reação de hipersensibilidade tipo IV, ou seja, mediada por linfócitos $\mathrm{T}$, responsável pela formação dos granulomas extraintestinais e vasculite cutânea, posteriormente com necrose e ulceração da pele sobrejacente. ${ }^{(20-23)}$ Recentemente, novas pesquisas tentam atribuir como causas a mutação da integrina beta-

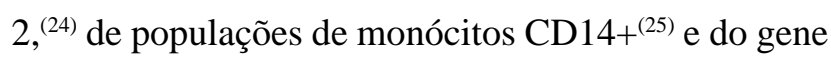
de reparo do DNA MLH-1. ${ }^{(26)}$

Histologicamente, a DCM se caracteriza por granulomas cutâneos não-caseosos, com células gigantes multinucleadas de Langhans e do tipo corpo estranho, os quais se distribuem na derme superficial, derme profunda e no tecido adiposo, similares aos granulomas do trato gastrointestinal. ${ }^{(1,2,4-7,9,12,16)}$ Outras características descritas são o infiltrado rico em eosinófilos e degeneração cística do colágeno (necrobiose). ${ }^{(1,5,5)}$

A DCM surge simultaneamente ou após o envolvimento gastrointestinal, e raramente o antecede. ${ }^{(1,2,7,11)}$ Ocorre principalmente quando há doença no intestino grosso, apesar de a doença de Crohn ser mais comum no intestino delgado. ${ }^{(1-3,5,6)} \mathrm{Em}$ adultos, a DCM geralmente surge após o diagnóstico da doença de Crohn em $70 \%$ dos casos, e raramente é relatada como sintoma inicial. Nas crianças, a doença de Crohn metastática surge simultaneamente aos sintomas gastrointestinais em quase metade dos casos. ${ }^{(1,2)} \mathrm{O}$ tempo de evolução e a gravidade das lesões cutâneas parecem evoluir em paralelo com a atividade da doença no trato gastrointestinal nos casos em que a doença de Crohn metastática surgiu simultaneamente ou sucedeu aos sintomas gastrointestinais. ${ }^{(4,15)}$

Ao exame físico, apresenta-se diferente de acordo com o local afetado. As lesões não-genitais podem variar de pápulas eritematosas a nódulos, placas ulceradas ou abscessos, enquanto as lesões genitais comumente se apresentam como edema e, às vezes, lesões ulceradas. ${ }^{(2,3,6,8,11,15,27)}$ Acomete principalmente os membros, particularmente as pernas, seguido por região inguinal, vulva, pênis, tronco, axila e face, em ordem decrescente. ${ }^{(1,3,5)}$ Também parece apresentar predileção por dobras cutâneas, tais como região inguinal, perineal e submamária. ${ }^{(3,12,13,15)} \mathrm{O}$ envolvimento genital parece ser mais comum nas crianças, ocorrendo em $85 \%$ dos casos. Nos adultos, as lesões mais frequentes são nódulos ou placas, com ou sem ulceração, nos braços e pernas, seguido por úlceras na região genital..$^{(1,2,4-6,27)}$

Devido suas características clínicas polimórficas, a DCM faz diagnóstico diferencial com diversas outras dermatoses, tais como: sarcoidose cutânea, infecção por micobactérias, infecções fúngicas, parasitoses, granulomatose de Wegener, síndrome de Behçet, histiocitose de células de Langhans, reação tipo corpo estranho, injeção factícia de substâncias (sílica, zircônio, berílio, tatuagens), hidradenite 
supurativa, linfedema, doenças sexualmente transmissíveis, reações alérgicas, síndromes paraneoplásicas, doença de Paget extramamária e hipertrofia de glândulas sebáceas. ${ }^{(1,2,4-9,27,16)}$

O diagnóstico da DCM consiste em biópsia da lesão cutânea, com presença de granulomas não caseosos ao exame anátomo-patológico. ${ }^{(2-4,8,9,12)}$ Caso necessário, poderá ser realizado cultura de tecidos, raio$\mathrm{X}$ de tórax e PPD para o diagnóstico diferencial. Na forma genital da DCM, é essencial excluir infecção por doenças sexualmente transmissíveis, tais como sífilis, linfogranuloma venéreo, granuloma inguinal e infecção crônica pelo vírus herpes simples. ${ }^{(2,4,8)}$

Apesar da resolução espontânea da doença de Crohn metastática ter sido descrita, este desfecho é incomum. ${ }^{(2,11)}$ A remoção cirúrgica do segmento intestinal afetado não necessariamente melhora a doença de Crohn metastática. ${ }^{(2,4)}$

Não há na literatura estudos clínicos randozimados e controlados sobre o tratamento da do- ença de Crohn metastática. Existem publicações relatando uso de metronidazol, , $^{(2,4,7,8,11,12,15,27)}$ corticoide oral ou tópico, ${ }^{(2,4,7,8,11,12,15,27)}$ azatioprina, ${ }^{(2,4,7,8,11,12,27)} 6$ mercaptopurina, ${ }^{(4,7,11)}$ ciclosporina, ${ }^{(2,4,15)}$ talidomida, ${ }^{(28)}$ sulfassalazina e seus derivados, $(2,4,7,8,11,27)$ tetraciclinas, ${ }^{(2,4,8,27)}$ desbridamento cirúrgico ${ }^{(7,11,12,15)} \mathrm{e}$ oxigenioterapia hiperbárica, ${ }^{(11,12,15)}$ porém com resultados isolados e controversos.

A terapia biológica com infliximabe, um anticorpo monoclonal quimérico anti-TNFá, tem sido usada para o tratamento das manifestações extra-intestinais da doença de Crohn..$^{(2,12,15)}$ Há seis publicações na literatura inglesa sobre pacientes com doença de Crohn metastática tratados com sucesso com uso do infliximabe..$^{(12,13,27,29-31)}$ Nosso paciente apresentou boa resposta à introdução da terapia biológica, com cicatrização completa das lesões.

Estudos controlados e randomizados serão necessários para determinar a eficácia e segurança do infliximabe na doença de Crohn metastática.

\begin{abstract}
The metastatic Crohn's disease involves granulomatous infiltration of skin in places anatomically separated from the gastrointestinal tract, with a tendency to chronicity. We report the case of male patient, 20 years old, complaining of pain and elimination of purulent secretion from ulcers in the perianal region, right inguinal and genital for six months, without improvement using metronidazole and ciprofloxacin. Previous proctocolectomy in 2002. On examination, the inspection revealed a fistulous orifice on posterior midline at $2.0 \mathrm{~cm}$ from the anal verge, an ulcer of $5.0 \mathrm{~cm}$ at the base of the scrotum and other ulcer encircling the base of the penis; on digital touch, anus fibrotic and incarcerated. Performed fistulotomy, biopsy and curettage of genital and inguinal ulcers. The histo-pathological study confirmed noncaseating granulomatous inflammatory process. Because of the failure of antimicrobial therapy, it was opted for treatment infliximab at the dose $5 \mathrm{mg} / \mathrm{kg}$ at weeks 0,2 and 6 , and azathioprine $2 \mathrm{mg} / \mathrm{kg} / \mathrm{day}$. In the end stage of induction, the patient had partial healing of ulcerated lesions, absence of secretion and pain relief. Currently in monitoring with outpatient infusions of infliximab every eight weeks.
\end{abstract}

Key words: Crohn's disease; Metastatic; Skin; Perineum; Biologic therapy.

\section{REFERÊNCIAS}

1. Emanuel PO, Phelps RG. Metastatic Crohn's disease: a histopathologic study of 12 cases. J Cutan Pathol 2008; 35: p. 457-461.

2. Palamaras I, El-Jabbour J, Pietropaolo N, Thomson P, Mann S, Robles W et al. Metastatic Crohn's disease: a review. JEADV 2008; 22: p. 1033-1043.

3. Balsamo F, Fraga JBP, Moreno WD, Formiga GJS. Doença de Crohn metastática para axila. Rev Bras Coloproct 2007; 27(1): p. 089-092

4. Gilson MR, Elston LC, Pruitt CA. Metastatic Crohn's disease: remisson induced by mesalamine and prednisone. J Am Acad Dermatol 1999; 41(3 Pt 1): p. 476-479.
5. Acker SM, Sahn EE, Rogers HC, Maize JC, Moscatello SA, Frick KA. Genital cutaneous Crohn disease: two cases with unusual clinical and histopathologic features in young men. Am J Dermatopathol 2000; 22(5): p. 443-446.

6. Schrodt BJ, Callen JP. Metastatic Crohn's disease presenting as chronic perivulvar and perirectal ulcerations in a adolescent pacient. Pediatrics 1999; 103(2): p. 500-502.

7. Martinez-Salamanca JI, Jara J, Miralles P, Berenguer J, Del Valle E, Hernandez C. Metastatic Crohn's disease: penile and escrotal involvement. Scand J Urol Nephrol 2004; 38(5): p. 436-437.

8. Macaya A, Marcoval J, Bordas X, Moreno A, Vasquez S, Peyri J. Crohn's disease presenting as prepuce and scrotal edema. J Am Acad Dermatol 2003; 49(2 Suppl Case Reports): p. S182-183. 
9. Goh M, Tekchandani AH, Wojno KJ, Faerber GJ. Metastatic Crohn's disease involving penile skin. J Urol 1998; 159(2): p. 506-507.

10. Crohn BB, Ginzburg L, Oppenheimer GD. Regional ileitis. JAMA 1932; 99: p. 1323-1329.

11. Dutheil P, Wallach D, Leibowitch M, Raynaud F, Dupont C. Metastatic cutaneous Crohn's disease in a child. J Pediatr Gastroenterol Nutr 1998; 26(3): p. 343-344.

12. Miller AM, Elliott PR, Fink R, Connell W. Rapid response of severe refractory metastatic Crohn's disease to infliximab. J Gastroenterol Hepatol 2001; 16: p. 940-942.

13. van Dullemen HM, de Jong E, Slors F, Tytgat GN, van Deventer SJ. Treatment of therapy-resistant perineal metastatic Crohn's disease after proctectomy using anti-tumor necrosis factor chimeric monoclonal antibody, cA2: report of two cases. Dis Colon Rectum 1998; 41: p. 98-102.

14. Poon KS, Gilks CB, Masterson JS. Metastatic Crohn's disease involving the genitalia. J Urol 2002; 167(6): p. 2541-2542.

15. Guest GD, Fink RL. Metastatic Crohn's disease: case report of an unusual variant and review of the literature. Dis Colon Rectum 2000; 43(12): p. 1764-1766.

16. Lehrnbecher T, Kontny HU, Jeschke R. Metastatic Crohn's disease in a 9-year-old boy. J Pediatr Gastroenterol Nutr 1999; 28(3): p. 321-323.

17. Parks AG, Morson BC, Pegum JS. Crohn's disease with cutaneous involvement. Proc R Soc Med 1965; 58: p. 241242.

18. Mountain JC. Cutaneous ulceration in Crohn's disease. Gut 1970; 11: p. 18-26.

19. Burgdorf W. Cutaneous manifestations of Crohn's disease. J Am Acad Dermatol 1981; 5: p. 689-695.

20. Slater DN, Waller PC, Reilly G. Cutaneous granulomatous vasculitis: presenting feature of Crohn's disease. J R Soc Med 1985; 78: p. 589-590.

21. Tatnall FM, Dodd HJ, Sarkany I. Crohn's disease with metastatic cutaneous involvement and granulomatous cheilitis. J R Soc Med 1987; 80: p. 49-51.

22. Shum DT, Guenther L. Metastatic Crohn's disease. Case report and review of the literature. Arch Dermatol 1990; 126: p. 645-648.
23. Coombs PRA, Gell PGH. Classification of allergic reactions responsible for clinical hypersensitivity and disease. In: Gell PGH, Coombs PRS, Lachmann, PJ, eds. Clinical Aspects of Immunology. Blackwell Scientific Publications Inc, Boston, Mass 1975: p. 761-781.

24. Bernstein CN, Sargent M, Gallatin WM. Beta2 integrin/ICAM expression in Crohn's disease. Clin Immunol Immunopathol 1998; 86: p. 147-160.

25. Grimm MC, Pavli P, Van de Pol E, Doe WF. Evidence for a CD14+ population of monocytes in inflammatory bowel disease mucosa - implications for pathogenesis. Clin Exp Immunol 1995; 100: p. 291-297.

26. Pokorny RM, Hofmeister A, Galandiuk S, Dietz AB, Cohen $\mathrm{ND}$, Niebergs HL. Crohn's disease and ulcerative colitis are associated with the DNA repair gene MLH1. Ann Surg 1997; 225: p. 718-723; discussion p. 723-725.

27. Escher JC, Stoof TJ, van Deventer SJ, van Furth AM. Successful treatment of metastatic Crohn disease with infliximab. J Pediatric Gastroenterol Nutr 2002; 34: p. 420423.

28. Kolivras A, De Maubeuge J, André J, Song M. Thalidomide in refractory vulvar ulcerations associated with Crohn's disease. Dermatology 2003; 206(4): p. 381-383.

29. Gill KR, Mahadevan U. Infliximab for the treatment of metastatic hepatic and pulmonary Crohn's disease. Inflamm Bowel Dis 2005; 11(2): p. 210-212.

30. Rispo A, Lembo G, Insabato L, Cozzolino A, Pesce G, Castiglione F. Successuful treatment of therapy-resistant metastatic Crohn's disease with infliximab. Br J Dermatol 2004; 150(5): p. 1045-1046.

31. Konrad A, Seibold F. Response of cutaneous Crohn's disease to infliximab and methotrexate. Dig Liver Dis 2003; 35: p. 351-356.

Endereço para Correspondência: GALDINO JOSÉ SITONIO FORMIGA

Rua Cônego Xavier, 276

Vila Heliópolis, São Paulo/SP

CEP: 04231-030

Telefone: (11) 2274-7600 (ramal 244)

E-mail: gformiga@ig.com.br 